\title{
Strain gradient drives shear banding in metallic glasses
}

Article in Physical Review B · September 2017

DOI: 10.1103/PhysRevB.96.094103

CITATIONS

0

4 authors, including:

Tian Zhili

Chinese Academy of Sciences

2 PUBLICATIONS 5 CITATIONS

SEE PROFILE
READS

83

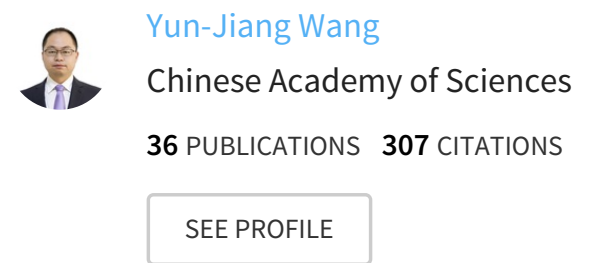

Some of the authors of this publication are also working on these related projects:

Project

Multi-time-scale computer simulations of the hierarchical dynamics in stress relaxation and creep of metallic glasses View project 


\title{
Strain gradient drives shear banding in metallic glasses
}

\author{
Zhi-Li Tian, Yun-Jiang Wang, Yan Chen, and Lan-Hong Dai* \\ State Key Laboratory of Nonlinear Mechanics, Institute of Mechanics, Chinese Academy of Sciences, Beijing 100190, \\ People's Republic of China \\ and School of Engineering Science, University of Chinese Academy of Sciences, Beijing 101408, People's Republic of China
}

(Received 4 November 2016; revised manuscript received 12 April 2017; published 6 September 2017)

\begin{abstract}
Shear banding is a nucleation-controlled process in metallic glasses (MGs) involving multiple temporal-spatial scales, which hinders a concrete understanding of its structural origin down to the atomic scale. Here, inspired by the morphology of composite materials, we propose a different perspective of MGs as a hard particle-reinforced material based on atomic-scale structural heterogeneity. The local stable structures indicated by a high level of local fivefold symmetry (L5FS) act as hard "particles" which are embedded in the relatively soft matrix. We demonstrate this concept by performing atomistic simulations of shear banding in CuZr MG. A shear band is prone to form in a sample with a high degree of L5FS which is slowly quenched from the liquid. An atomic-scale analysis on strain and the structural evolution reveals that it is the strain gradient effect that has originated from structural heterogeneity that facilitates shear transformation zones (STZs) to mature shear bands. An artificial composite model with a high degree of strain gradient, generated by inserting hard MG strips into a soft MG matrix, demonstrates a great propensity for shear banding. It therefore confirms the critical role strain gradient plays in shear banding. The strain gradient effect on shear banding is further quantified with a continuum model and a mechanical instability analysis. These physical insights might highlight the strain gradient as the hidden driving force in transforming STZs into shear bands in MGs.
\end{abstract}

DOI: 10.1103/PhysRevB.96.094103

\section{INTRODUCTION}

Shear banding is a ubiquitous phenomenon in a wide variety of materials including metals, polymers, the Earth's mantle, and granular solids [1-3]. It remains as a fundamental and intriguing topic in condensed matter physics, glassy physics, and materials science. Especially in the case of metallic glasses (MGs), the complexity of the disordered structure makes it very complicated to find a direct correlation between the emergence of shear bands and its deep structural origin [3-7]. Although short- and medium-range order may exist in MGs [810], the lack of a long-range atomic periodicity leads to inherent nanoscale structural heterogeneity. Both experiments [11$16]$ and atomic simulations $[4,17-19]$ have verified structural heterogeneity in MGs. Pioneering works have described this heterogeneity as solidlike and liquidlike regions [12,20-22]. Liquidlike regions have a lower atomic packing density than solidlike regions and thus are fertile regions for shear transformation zones (STZs) [23,24], which are commonly hypothesized as the plastic carriers of MGs. Because of the alternating distribution of solidlike and liquidlike regions, the elastic and inelastic responses of the two regions under external stimuli will eventually result in an inhomogeneous deformation field [25-27]. Moreover, from the perspective of the potential energy landscape (PEL) [28,29], the probability of hopping between the neighboring "subbasins" in liquidlike regions is larger than that in solidlike regions. It demonstrates that the trigger mechanisms of STZs and the corresponding relaxation rely heavily on structural heterogeneity [30-32]. In the past decades, many insightful physical concepts have been proposed, e.g., free volume [33], STZs [23,24], the cooperative shear model (CSM) [34], soft spots [18], and flow units [35],

*Corresponding author: 1hdai@1nm.imech.ac.cn all of which are based on liquidlike regions. However, apart from liquidlike regions, we may also incorporate the other side of the structural heterogeneity, i.e., solidlike regions, into an understanding of the shear banding phenomenon. The percolation of stable structures in solidlike regions has been found to affect strain localization [36]. A key question then arises as to how the structural heterogeneity of solidlike and liquidlike regions cooperatively promotes the emergence of shear bands.

As mentioned above, it is probably the combined effect of both solidlike and liquidlike regions that synthetically promotes the emergence of shear bands. Besides the $\sim 25 \%$ liquidlike regions [12], the remaining solidlike regions are also heterogeneous $[11,18]$. A Voronoi index [37,38] in the form of $\left\langle n_{3}, n_{4}, n_{5}, n_{6}, \ldots, n_{i}, \ldots\right\rangle$ is widely adopted to characterize the local structure packing, where $n_{i}$ denotes the number of $i$-edge polygons. Herein the local fivefold symmetry (L5FS), defined as the fraction of pentagons $\left(n_{5} / \sum_{i} n_{i}\right)$ in each local cluster, is quantified as the structural indicator to characterize stable structures in MGs [39-45]. Figure 1(a) shows that the high L5FS regions are embedded into the matrix with medium and low degrees of L5FS. Inspired by particle-reinforced metal matrix composites [46], we may reasonably hypothesize that there exist hard "particles" which represent the most stable clusters showing a high structural and energetic stability, as schematically shown in Fig. 1(b). It should be emphasized that MGs have a continuous distribution of atomic packing patterns $[14,47,48]$. They should not be misunderstood as real particle-reinforced composite materials. But this operation does facilitate the analysis of the intrinsic origins of the versatile deformation modes in MGs. Because of the heterogeneous microstructure, the stress or deformation response of a local region depends not only on its own state, but it is also involved with the states of the surrounding environments, just as was proposed by the nonlocal continuum 

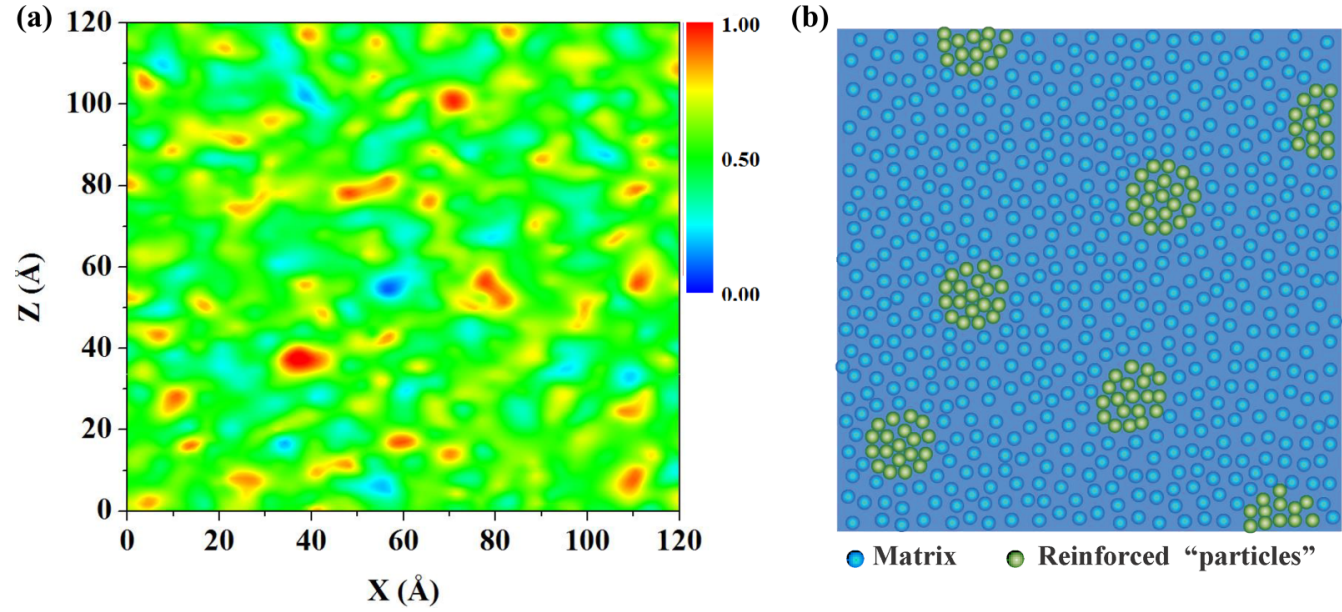

FIG. 1. MG as a particle-reinforced composite material. (a) The spatially inhomogeneous distribution of the level of L5FS. A slice of $5 \AA$ along the $y$ direction is coarse-grained for the plot. (b) Schematic illustrations of the structural heterogeneity and the proposed composite mode of MGs.

field theory [49]. The strain gradient is such a physical quantity characterizing the limited nonlocality and it has already taken the spatial distribution of the strain field into consideration. The influences of strain gradient on strengthening, work hardening, and shear instability were broadly reported before [46,50-56]. The strain gradient is highly anticipated to provide the long time missing bridge linking the activation of isolated STZs to the emergence of shear bands.

In this paper, we endeavor to line up atomic-scale structural heterogeneity to shear banding in MGs. We consider stable clusters with a high degree of L5FS as the hard "particles" which are capable of resisting deformation and consequently deal with MGs as particle-reinforced metal matrix composites. This operation facilitates revealing the strain gradient due to structural heterogeneity as the hidden factor driving shear banding in MGs. This finding may also rationalize the simulation informed, seemingly counterintuitive, enhanced shear banding susceptibility in slowly quenched thermodynamically stable MG structures.

\section{METHODS}

The simulations are performed with the LAMMPS code [57]. A commonly accepted Finnis-Sinclair type embedded atom method (EAM) potential is adopted to describe the atomic interactions [58]. We design two series of samples to carry on compression and shear, respectively. The first four samples to be compressed consist of 388800 atoms with dimensions of $\sim 37.4 \mathrm{~nm} \times 74.8 \mathrm{~nm} \times 2.5 \mathrm{~nm}$. The other four samples to be sheared consist of 122880 atoms with dimensions of $\sim 16.5 \mathrm{~nm} \times 10.0 \mathrm{~nm} \times 13.3 \mathrm{~nm}$. The temperature and pressure are controlled with a Nosé-Hoover thermostat [2] and Parrinello-Rahman barostat [3], respectively. Period boundary conditions are used during the sample preparation process. In the compression test, a strain rate of $10^{9} \mathrm{~s}^{-1}$ is applied in the $y$ direction at $100 \mathrm{~K}$ while the $x$-direction boundary is kept free and periodic boundary conditions are used for $y$ and $z$ directions. Shear deformation is also carried out at $100 \mathrm{~K}$ with the same strain rate of $10^{9} \mathrm{~s}^{-1}$, but all three dimensions are periodic. To prepare different initial structures, we generate
$\mathrm{Cu}_{50} \mathrm{Zr}_{50}$ MG samples in molecular dynamics (MD) by setting four different cooling rates, i.e., 0.02, 0.2, 2, and $20 \mathrm{~K} / \mathrm{ps}$, respectively. Local atomic rearrangement is characterized by the local von Mises strain $\eta^{\text {Mises }}$ [59].

\section{RESULTS AND DISCUSSION}

\section{A. Metallic glass as hypothetical particle-reinforced composite}

As demonstrated in Fig. 1, we strategically hypothesize MG as a particle-reinforced composite material to mimic its microscopically heterogeneous structure. We measure the average L5FS [Fig. 2(a)] in the four samples as well as other symmetries such as L3FS, L4FS, L6FS, and L7FS (Fig. S1 [60]). Only L5FS increases when quenched from the liquid to the solid state, while the other symmetries decrease to a very low level. L5FS also has a sharp increase near the glass transition temperature. Moreover, Fig. S2 [60] shows that L5FS not only has a dominating fraction but also shows the most inhomogeneous distribution, as demonstrated by the standard deviation. Therefore, L5FS is the prominent structure with a solidlike feature while the other symmetries are possibly related to the liquidlike features [40,41]. The slower the cooling rate is, the more the L5FS appears in the as-quenched structure. The results of the potential energy change shown in the inset of Fig. 2(a) and the radical distribution function (RDF) (Fig. S3[60]) also confirm that a slow cooling rate yields an energetically stable structure. Although samples prepared with different cooling rates do show some differences in the L5FS percentage, such differences are very small. A further analysis of the statistical distribution of L5FS shows that a slower cooling rate results in a more inhomogeneous distribution of L5FS; see Fig. 2(b). We will show that shear banding is more sensitive to the distribution of L5FS than its absolute fraction. Figure 2(c) gives some typical clusters from the highest degree of L5FS to the relatively lower ones.

Generally, samples with more hard "particles" are usually thought to be more resistant to deformation. However, we find that samples with more stable L5FS are more susceptible to shear banding, while the samples with less L5FS deform 

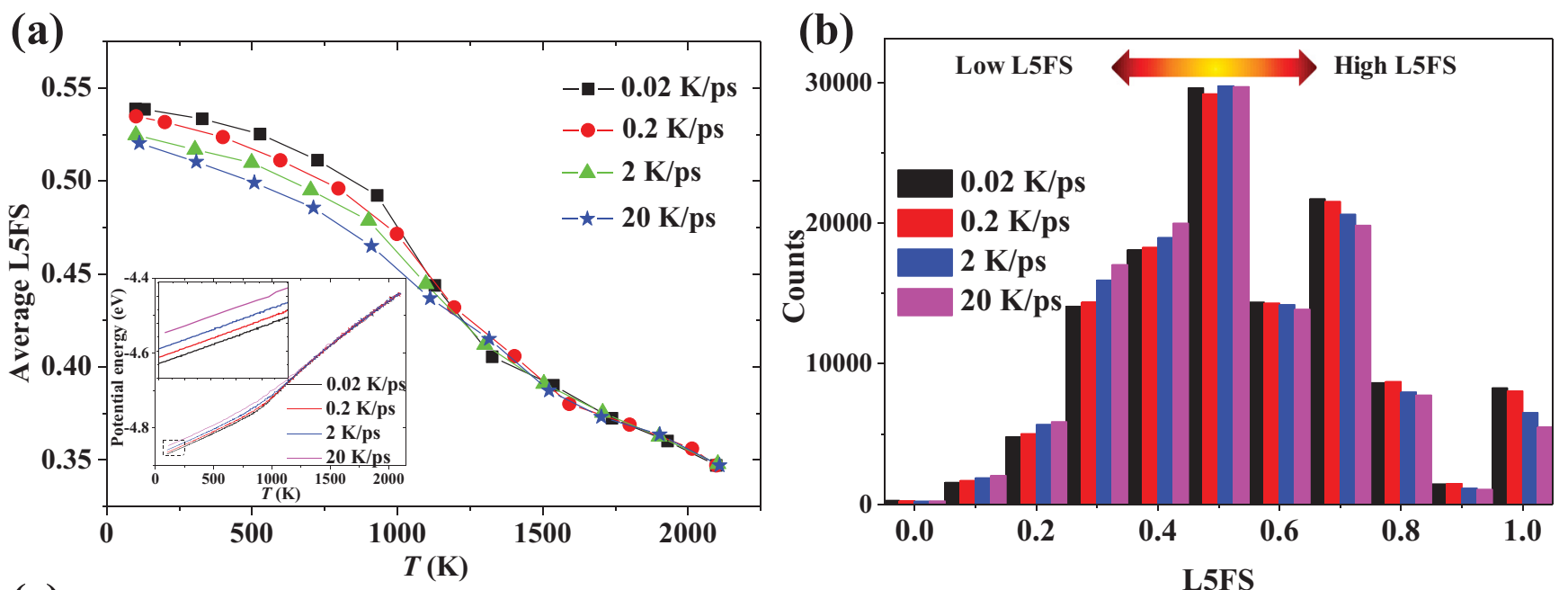

(c)
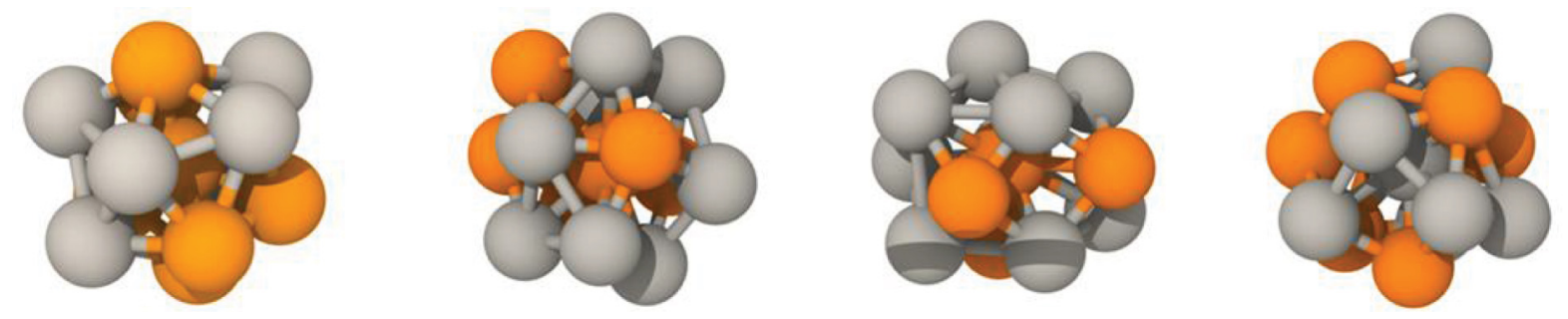

$<0,0,12,0>$, L5FS $=1.00$

$<0,1,10,2>$, L5FS $=0.77$

$<0,2,8,2>, \mathrm{L5FS}=0.67$

$<0,3,6,4>$, L5FS $=0.46$

FIG. 2. Features of the MG structure characterized by L5FS. (a) Evolution of the average L5FS during cooling with different cooling rates. The inset is the evolution of potential energy and its closeup view at the final states. (b) Statistical distribution of L5FS in four samples prepared with different cooling rates. A slower cooling rate leads to more structural heterogeneity. (c) Some typical clusters from the highest degree of L5FS to the relatively lower ones. The orange and gray spheres represent $\mathrm{Cu}$ and $\mathrm{Zr}$ atoms, respectively.

homogeneously; see Fig. 3(a) (see compression in Fig. S4 [60]). The color scheme is the local atomic shear strain $\eta^{\text {Mises }}$ [59]. Here, we take L5FS $>0.70$ as a threshold for the atoms in the hard particles. To break down the different mechanical properties between the hard particles and the matrix, we extract the stress-strain curves of these regions, respectively, in Fig. 3(b). The atomic shear stress is an average of the von Mises stress of specified atoms. Apparently, we see the modulus of hard particles is obviously larger than that of the others. We further trace the specified atoms which have undergone a large deformation ( $\left.\eta^{\text {Mises }} \geqslant 0.2\right)$ and observe the structural change of the "adjoining" atoms with the same atom identification from the very beginning. As Fig. 3(c) shows, the mean atomic shear strain of the traced atoms and the other ones are synchronous at the beginning but separate once a shear band appears. The L5FS evolutions of the traced atoms and the others follow the same rule [Fig. 3(d)]. Before the emergence of shear bands, L5FS of the traced atoms changes slightly and it starts to decrease quickly as the atomic strain begins to increase, while the L5FS of the other atoms remains almost at the same level during the whole process. This indicates that high L5FS areas do not experience a significant atomic rearrangement until the accumulated deformation is so large that they must bear extra deformations, which in turn decreases the L5FS fraction [Fig. 3(f)]. With the hypothesis that the hard particles are homogeneously distributed throughout the entire sample [see Fig. 1(b)], the interval space $\lambda$ of the hard particles could be derived as $\lambda=\frac{1}{2} d_{p}\left(\sqrt{\frac{2 \pi}{3 f_{p}}}-\frac{4}{\pi}\right)$ [52], where $f_{p}$ is the fraction of hard particles and $d_{p}$ is the average size of the hard particles $(\sim 10 \AA$ A based on Fig. 1). We calculate the average strain gradient as $\gamma / \lambda$, where $\gamma$ is the macroscopic shear strain. As Fig. 3(e) shows, the strain gradient effect is magnified step by step and the samples with the largest degree of L5FS show the most pronounced strain gradient effect. All of this information reminds us that the inherent strain gradient due to structural heterogeneity could be a candidate for the driving force for the initiation of shear banding, as has been shown in particle-reinforced metal matrix composites $[46,52,61]$.

\section{B. Strain gradient effect}

To clarify the effect of hard particles on STZ activation [62], we examine the correlation between the distribution of L5FS and that of atomic strain at the early stage of deformation, as well as the moment just before shear localization. In the early stage, STZs are mostly activated in low L5FS regions which are surrounded by higher L5FS regions [Fig. 4(a)]. As deformation goes on, STZ activation regions are broadened but are still in localized regions until the skeleton of the high degree of L5FS can no longer bear such a large deformation. Finally, the activated regions coalesce and we may see deformation developing in some specific directions that eventually leads to the formation of shear bands [Fig. 4(b)]. It is easy to find that hard particle regions and the matrix carry on different 


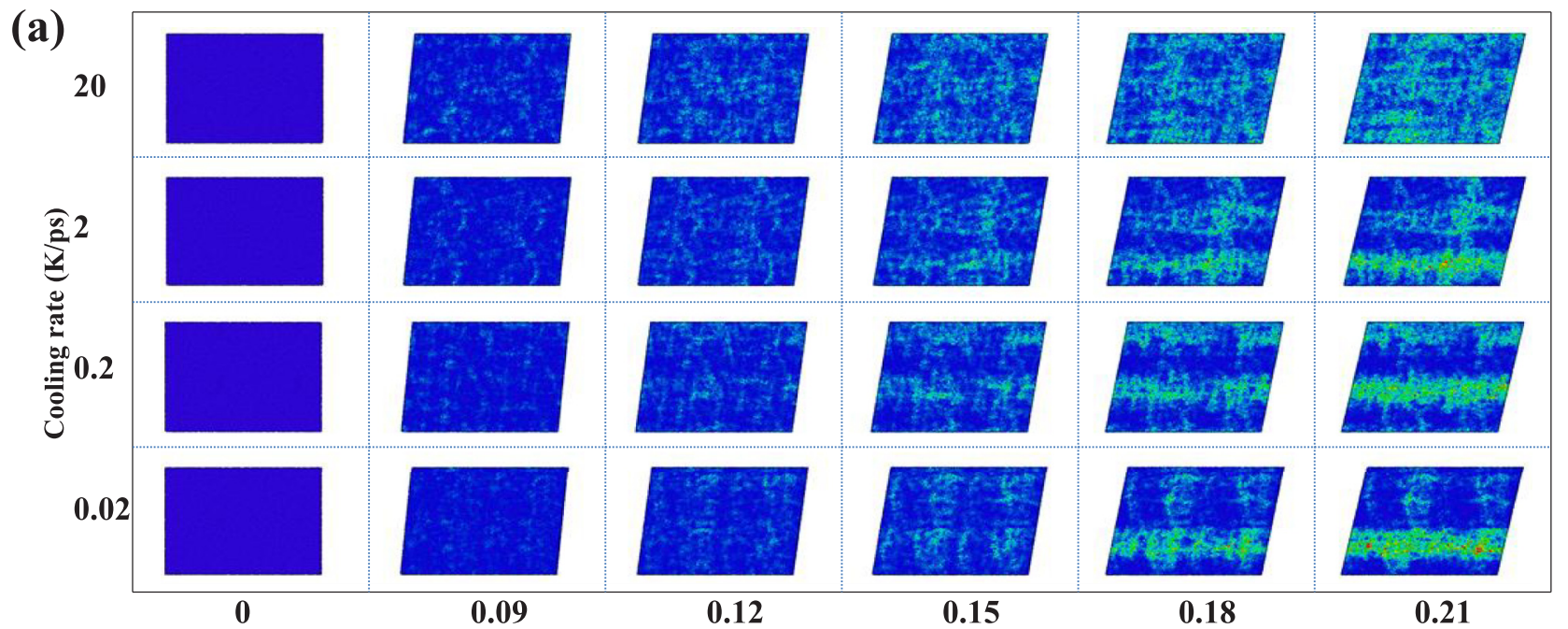

Macroscopic Strain

(b)

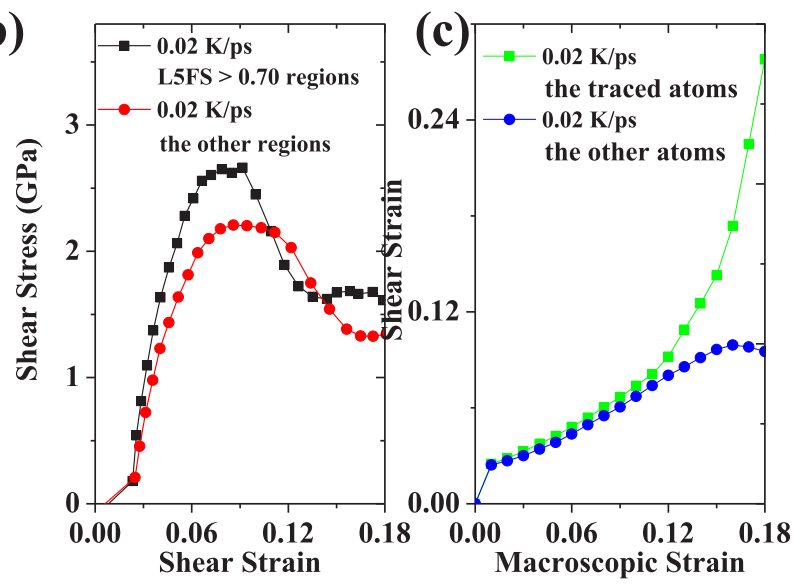

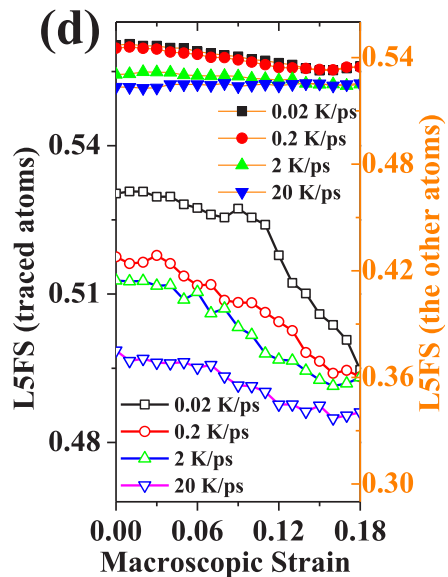

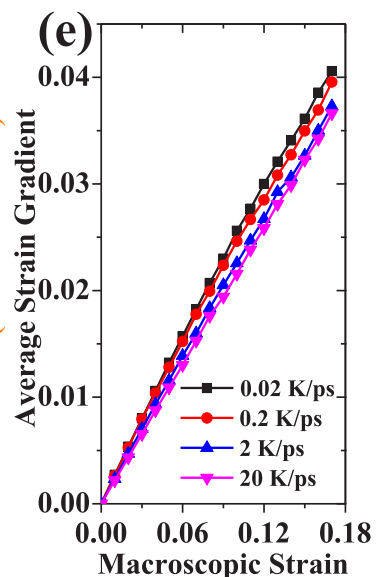

(f)

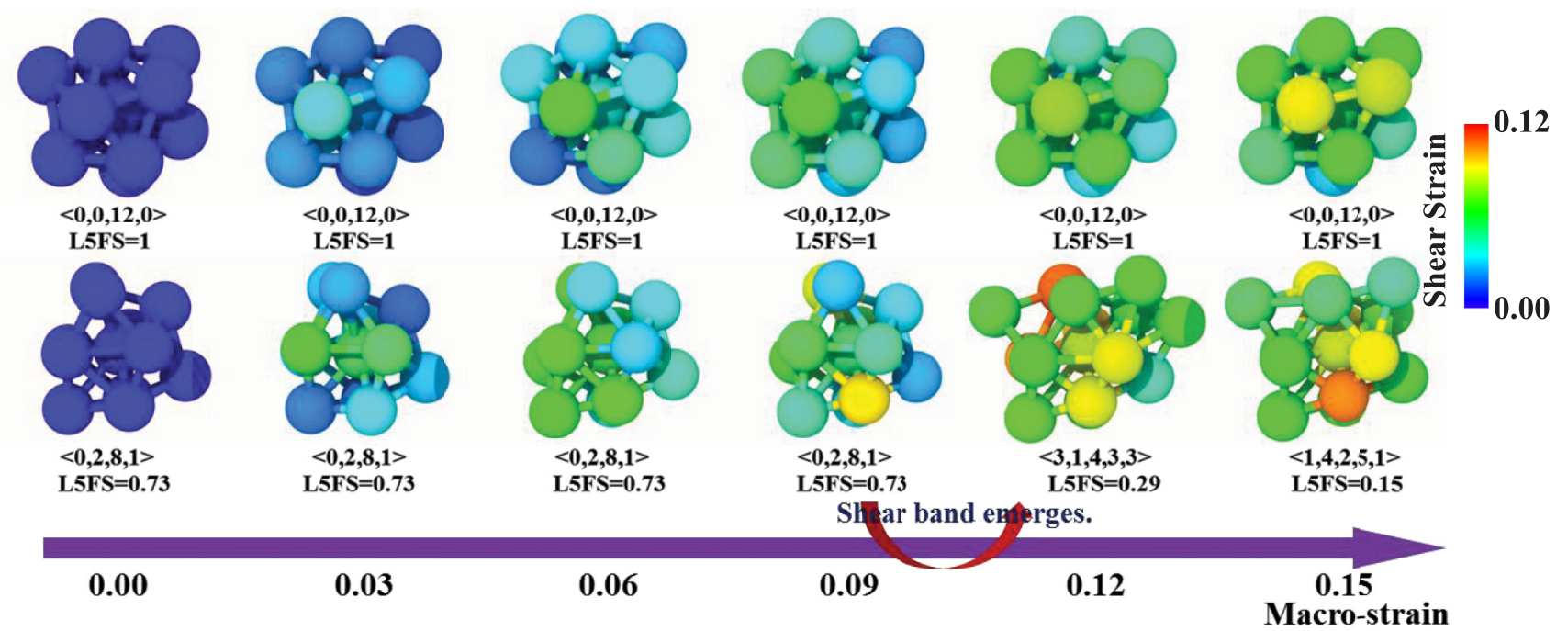

FIG. 3. Deformation pattern and the structural evolution during shear. (a) Shear deformation patterns of the samples with different cooling rates. (b) Local mean atomic shear stress vs atomic shear strain for the hard particles and the other regions. (c) Average atomic shear strain of the traced atoms and the other atoms; the traced atoms are those that experienced atomic shear strain larger than 0.20 after shear banding. (d) Variation of L5FS of the traced atoms and the other atoms. (e) Average strain gradient in samples with different cooling rates vs macroscopic strain. (f) Evolution of the clusters with the highest degree of L5FS (L5FS $=1$ ) and a relatively low degree of L5FS (L5FS $=0.73$ ). 

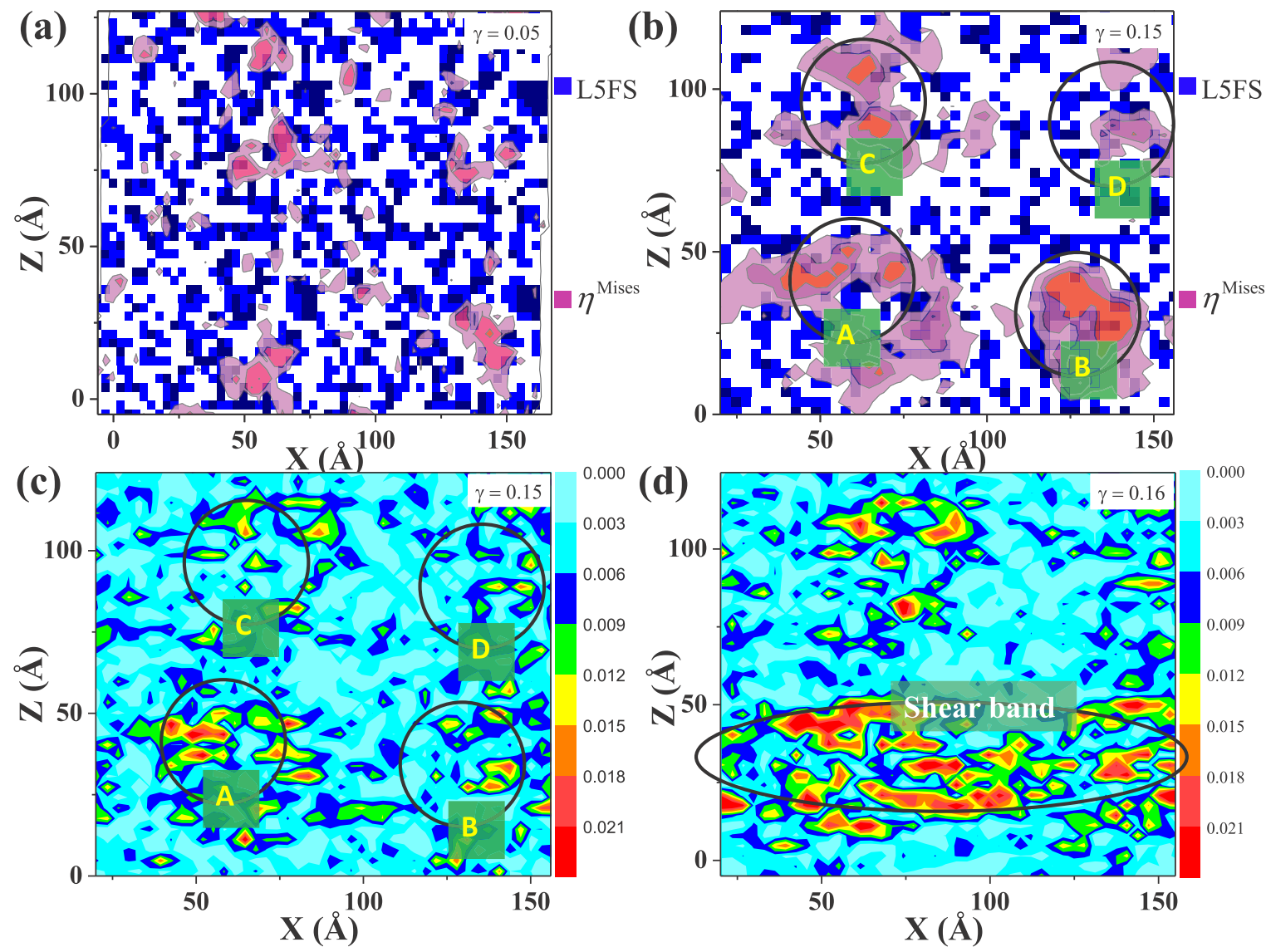

FIG. 4. The role of strain gradient played in driving shear banding. (a) Deformation pattern at $\gamma=0.05$ (before yielding). (b) The moment right before the formation of shear bands at $\gamma=0.15$. Percolation channels of STZs initially avoid high L5FS regions, but it can even destroy these strong L5FS regions to form shear bands with larger strain. The blue squares represent higher values of L5FS, while the blank areas mean low; pink and red colors show the local deformation contours. (c) Strain gradient field in the $z$ direction right before the emergence of a shear band, which corresponds to the deformation field as shown in (c). The circles A, B, C, and D label the same regions in (b) and (c). (d) Strain gradient field in the $z$ direction right after the formation of a shear band. The ellipsoid corresponds to the shear banding region.

magnitudes of strain and an obvious strain gradient therefore comes into effect. The strain gradient promotes the activation of STZs within the region between the neighboring hard particles.

To quantify the strain gradient effect, we provide its field to compare with the evolution of the structural features. We coarse-grain the simulation cell with small boxes at a resolution of $\delta=3 \AA$ and do an average of the atomic shear strain in the small boxes perpendicular to the shear plane $(x z$ plane). Then we can derive the strain gradient between the adjacent small boxes according to $\xi_{i, z}=\frac{\gamma_{i+1, z}-\gamma_{i, z}}{\lambda}$, where $i$ means the index of the small boxes and $z$ denotes the specified direction. We find a very good correspondence between the strain gradient fields [Fig. 4(c)] and the deformation [Fig. 4(b)]. Local regions where strain has accumulated penetrate into each other where the strain gradient effect is apparent, as shown in the regions A-D marked in both Figs. 4(b) and 4(c). Once the shear band sets in [Fig. 4(d)], much of the large strain gradient locates inside of the shear band. It indicates that the strain gradient does play a significant role in the nucleation pathway of shear bands [61]. Recently, we noted that similar ideas involving stress fields to account for the shear banding of MGs have also been proposed [63].
To more intuitively and straightforwardly demonstrate the critical effect of hard particles on the emergence of shear bands, we prepare an artificial composite model by inserting small, slowly quenched $(0.02 \mathrm{~K} / \mathrm{ps}) \mathrm{MG}$ strips into a fast-quenched (20 K/ps) sample [Fig. 5(a)]. In this way, we introduce more L5FS into the fast-quenched samples. This operation results in a higher strain gradient due to enhanced structural heterogeneity. After thermal relaxation at $500 \mathrm{~K}$ for $1 \mathrm{~ns}$ to eliminate the artificial boundary effect, the model is sheared at the same strain rate as the fast-quenched sample $(20 \mathrm{~K} / \mathrm{ps})$. It shows that the composite model presents strong shear localization [Fig. 5(c)] instead of homogeneous deformation [Fig. 5(b)]. The result further confirms the idea that the strain gradient originating from structural heterogeneity drives shear banding [61].

\section{Correlation between atomic strain}

We also calculate the two-time correlation [64] of the atomic shear strain to show the dependence of deformation on structure. The correlation function is defined as $C=$ $\left\langle\eta_{\gamma_{1}}(\mathbf{r}) \cdot \eta_{\gamma_{2}}(\mathbf{r})\right\rangle$, where $\eta_{\gamma}(\mathbf{r})$ represents the atomic shear strain 
(a)
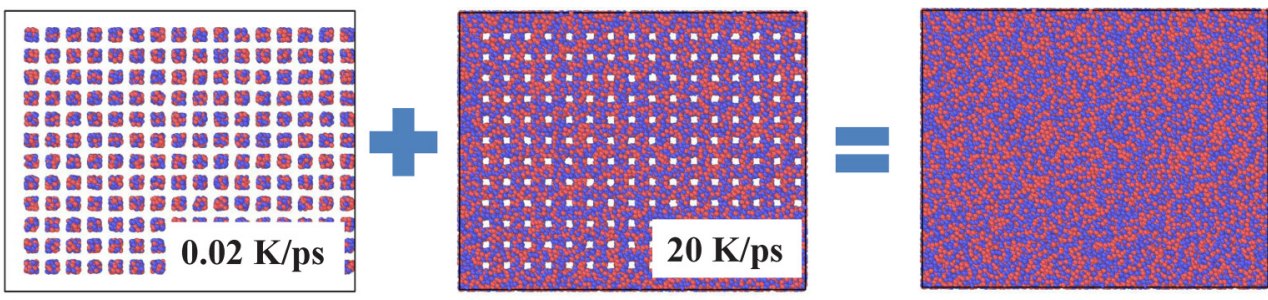

(b)

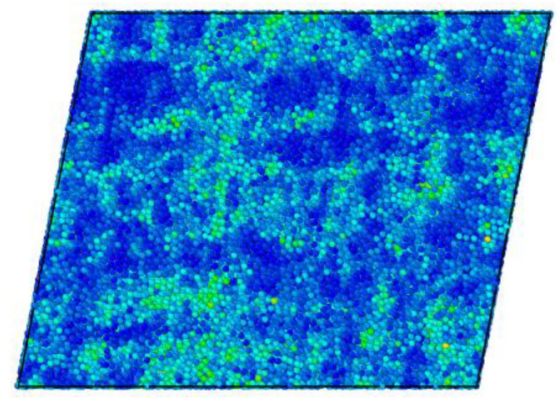

$20 \mathrm{~K} / \mathrm{ps}$ fast quenched model

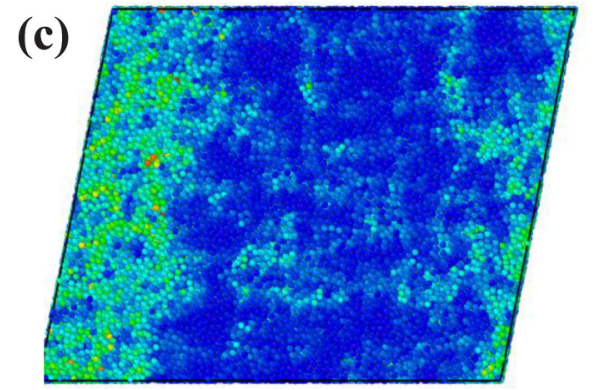

Artificial composite model

FIG. 5. Hypothesized composite model supporting the crucial role of the strain gradient in driving the shear band. (a) Sketch of the design of the composite model with enhanced structural heterogeneity and the associated strain gradient effect. (b) Deformation patterns of the fast-quenched sample and (c) the composite model with an extra level of strain gradient.

at a distance of $\mathbf{r}$ and at the macroscopic shear strain $\gamma$. Based on this definition, we analyze the dependence of deformation on the initial structure by setting the configuration of $\gamma_{1}=0.01$ as the reference [Fig. 6(a)]. It shows that the correlation decreases slowly in the early stage while it decays very fast once a shear band forms. This is because L5FS causes different degrees of the "cage effect" in the samples with different fractions and distributions of stable structures. In the early stage, high L5FS indicates a high resistance to STZ activation and therefore leads to a higher correlation with the previous deformation. Once a shear band appears, the correlation abruptly decreases, which means that steady-state shear flow starts and the correlation between deformation and structure is lost. There is an obvious transition when the local deformed regions penetrate into each other. The most pronounced L5FS sample shows the highest correlation because of the strong constraint effect of the stable structure. We can also define a susceptibility factor which indicates the accumulated effect of deformation on the structure by setting $\gamma_{1}$ and $\gamma_{2}$ as flowing references [Fig. 6(b)]. The susceptibility factor is the response function of local strain. We find that the susceptibility factor is amplified during loading. It shows a very different dynamic process before and after the transition at the initiation of a shear band. Before the shear bands form, we see a lower value in the higher L5FS sample. This shows the constraint effect of the stable structure. Rearrangement is suppressed mainly in the regions surrounded by hard particles and thus leads to a higher strain gradient over there. The higher strain gradient further promotes strain localization. Once the shear band forms, the susceptibility factor increases quite fast in the sample with a higher degree of L5FS. It means that the accumulated effect or strain gradient effect is so large that the system loses its stability. At this moment when the skeleton of the stable structure is broken, localized deformed regions connect with each other and form a larger scale strain localization.
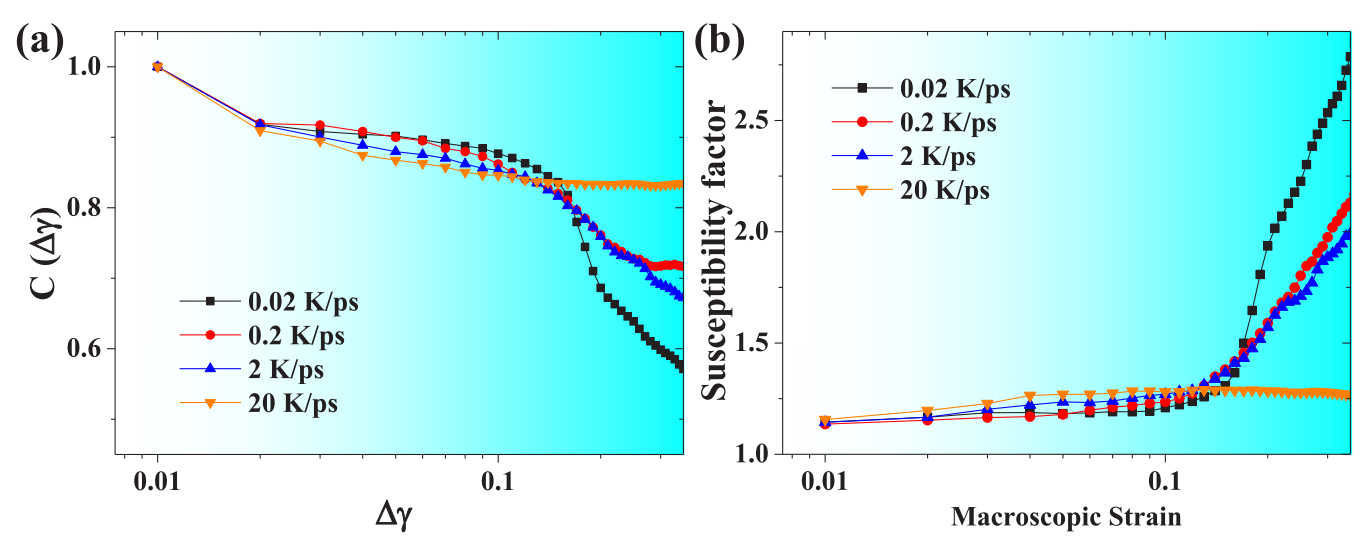

FIG. 6. (a) Two-time correlation of the atomic shear strain as a function of the strain interval. (b) Susceptibility factor with flow reference as a function of macroscopic strain. 

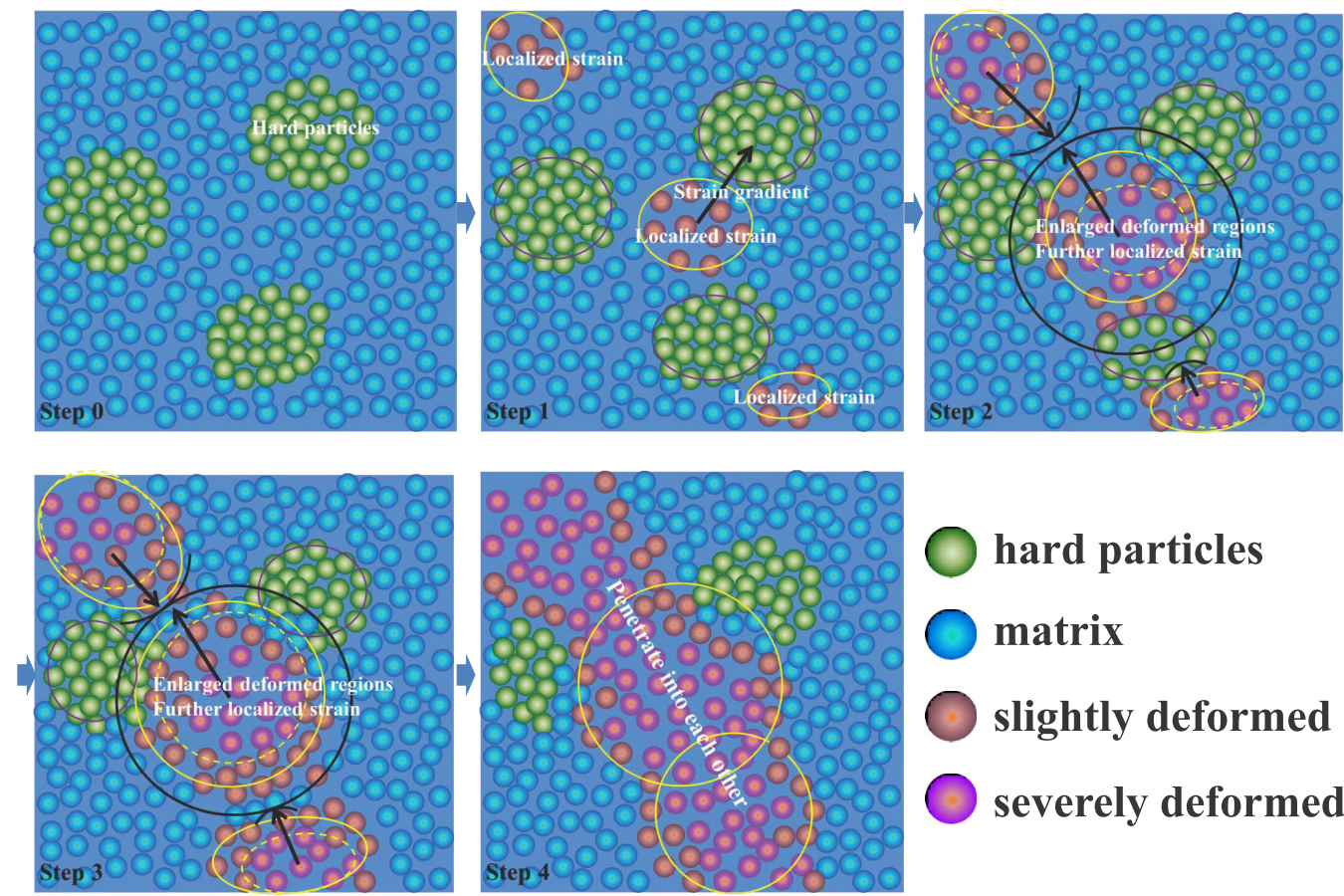

\section{hard particles}

matrix

slightly deformed

severely deformed

FIG. 7. A schematic demonstration of the self-feedback process of shear banding in metallic glasses which involves a critical role of strain gradient due to inherent structural heterogeneity.

\section{Scenario of strain gradient in driving shear banding}

Actually, the strain gradient effect which originates from structural heterogeneity comes into effect by inhomogeneous energy dissipation. The highly deformed regions in the liquidlike structures dissipate more energy while hard particle regions in the solidlike structures strengthen the material but dissipate less energy. The hard particles function as obstacles hindering the motion of STZs, which leads to enhanced hardening (Fig. S5 [60]) and more localized deformation. It is similar to that of geometrically necessary dislocations which are blocked by some defects and further lead to work hardening in crystals $[50,54,56,65]$. Because of the existence of hard particles, activated regions cannot be easily broadened. In other words, activated regions are blocked in the regions surrounded by a high degree of L5FS regions. In this sense, we say that the hardening behavior of the strain gradient effect is similar between amorphous and crystal materials. The inhomogeneity of energy dissipation further aggravates the strain gradient. The self-feedback process (Fig. 7) between the strain gradient and inhomogeneous energy dissipation finally reaches a limit, at which point the skeleton of MG cannot bear further deformation. Many localized regions start to yield and then connect to each other as strain continues. Then shear bands form in the direction of maximum stress and finally the whole system collapses.

\section{E. Continuum model and shear stability analysis}

To demonstrate quantitatively the role of strain gradient in promoting the formation of shear bands, a mechanismbased continuum mechanics model is built (see details in the Supplemental Material [60]). To simplify the problem, we consider a simple shear problem [66,67]. The governing equations of the system are shown as follows:

$$
\begin{gathered}
\tau=f(\chi, \gamma, \dot{\gamma}), \\
\frac{\partial^{2} \tau}{\partial y^{2}}=\rho \frac{\partial^{2} \gamma}{\partial t^{2}}, \\
\dot{\chi}=\frac{F(\tau) \exp (-1 / \chi) \tau}{c_{0}}\left[1-\frac{\chi}{\hat{\chi}(\dot{\gamma})}\right]+D \frac{\partial^{2} \chi}{\partial y^{2}},
\end{gathered}
$$

where

$$
F(\tau)=2 \varepsilon_{0} R_{0} \exp \left(-\frac{W\left(f_{p}\right)}{k_{B} T}\right) \cosh \left(\frac{\tau \Omega / 2}{k_{B} T}\right)\left(1-\frac{1}{\tau}\right) .
$$

Equation (1) is the constitutive law where we assume that the shear stress $\tau$ is dependent on strain $\gamma$, strain rate $\dot{\gamma}$, and effective disorder temperature $\chi$. The concept of effective disorder temperature [68-72] is defined to describe the configuration order change during deformation. Equation (2) is the momentum equation where $\rho$ is the mass density. Equation (3) is the evolution of effective disorder temperature where $c_{0}, R_{0}, \varepsilon_{0}, \tau_{y}$, $k_{B}, T$, and $D$ are the effective temperature specific heat, transformation rate constant, strain accumulated per STZ reversal, yield stress, Boltzmann constant, kinetic-vibrational temperature, and diffusion coefficient, respectively. $\hat{\chi}(\dot{\gamma})$ is the steadystate value of the effective temperature which is a function of strain rate as $\hat{\chi}(\dot{\gamma})=\frac{\chi_{w}}{\log \left(\frac{q_{0}}{t_{0} \dot{\gamma}}\right)}[73,74]$. Parameters include the effective temperature activation barrier $\chi_{w}$ and the nondimensional strain rate where STZ theory breaks down at $q_{0}$.

It is noted that the strain gradient term is implicitly included in the rate switching function which describes the rate at which 


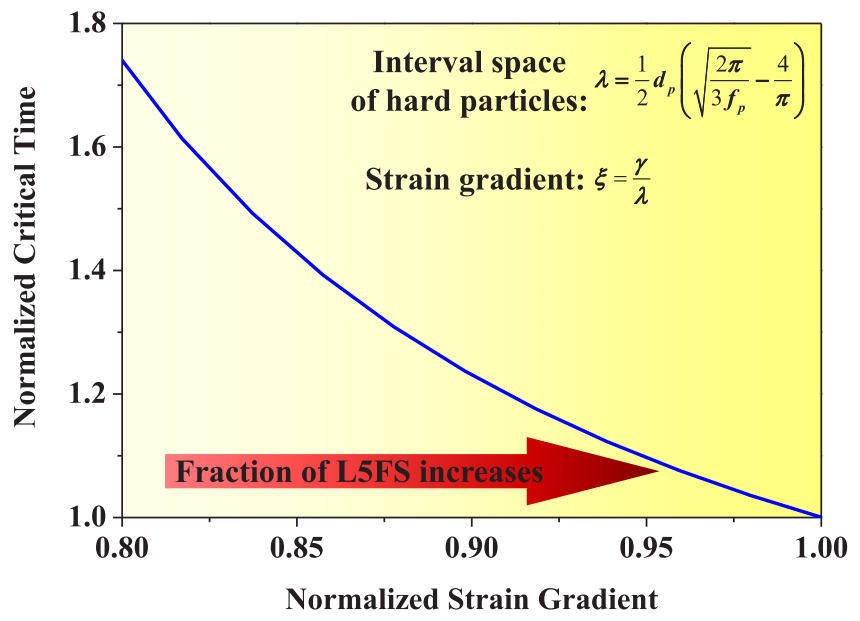

FIG. 8. Normalized characteristic time of instability as a function of the normalized strain gradient.

STZ reversals take place in response to applied shear stress. Due to structural heterogeneity, the rate switching function is related to the activation energy term $W\left(f_{p}\right)$, which further builds the connection with the volume fraction $f_{p}$. Thus the strain gradient is involved in $W\left(f_{p}\right)$ since strain gradient can be measured by $\frac{\gamma}{\lambda}$, in which the spacing of hard particles $\lambda$ has a relation with the volume fraction $f_{p}$ as $\lambda=\frac{1}{2} d_{p}\left(\sqrt{\frac{2 \pi}{3 f_{p}}}-\frac{4}{\pi}\right)$. $d_{p}$ is the average size of the hard particles. In our simulations, the slowest quenched samples have the highest volume fraction of L5FS and thus show the most apparent strain gradient effect.

According to Johnson and Samwer [62], the activation energy of STZ can be described by $W\left(f_{p}\right)=\frac{8}{\pi^{2}} \mu\left(f_{p}\right) \gamma_{c}^{2} \varsigma \Omega$, where $\mu$ is the shear modulus which is related to the volume fraction $f_{p}$ of the hard particles, $\gamma_{c}$ is the critical strain, $\varsigma$ is the correction factor, and $\Omega$ is the volume of a STZ. Considering the clusters with a high degree of L5FS as inclusions, we can get the effective modulus via the self-consistent model $[75,76]$. The modulus is the effective modulus or an average modulus of the whole sample which mainly reflects the cooperative effect of both the matrix and hard particles. The activation energy of the weak spots may not vary much since it is located in regions with a low degree of L5FS. The activation energy of the hard particles is high compared to liquidlike regions, which leads to strain accumulation in liquidlike regions and further results in an intense strain gradient effect.

After performing an instability analysis on the governing equations, we can obtain the onset condition of shear instability as follows:

$$
\frac{\frac{F(\tau) \exp (-1 / \chi) \tau}{c_{0} \hat{\chi}}\left(\frac{1}{\chi_{w}}-1\right)}{D k^{2}}>1 .
$$

This instability criterion is similar to the free volume instability criterion [77,78]. The numerator and denominator terms mean the net creation and diffusion of effective temperature, respectively. The underlying physics is such that perturbation will grow with time and shear band instability sets in only if the net creation of the effective temperature is faster than its diffusion. The stability of this system is controlled by the competition between these two processes. Here, the strain gradient is involved in the creation of the effective temperature.
We can also find the fastest mode for shear instability growth which corresponds to a maximum of growth rate $\alpha$. Thus we obtain the relation between the characteristic time corresponding to the fastest shear instability mode as follows:

$$
t_{c}=\frac{c_{0} \hat{\chi}(\dot{\gamma})}{F(\tau) \exp (-1 / \chi) \tau\left(1 / \chi_{w}-1\right)} .
$$

Here, the characteristic instability time characterizes how fast the perturbations grow and the system loses stability. A small characteristic time means it is easy to form a shear band in the system. As Fig. 8 shows, we find that the higher the strain gradient is, the shorter is the critical time. This means that it is the strain gradient that drives the system to lose mechanical stability and leads to the emergence of shear bands. Also, the strain gradient comes from inherent structural heterogeneity which is characterized by different degrees of L5FS.

\section{CONCLUSIONS}

In conclusion, we propose a different perspective on the correction between the strain gradient and shear banding in MG with a demonstration of atomistic simulations. To clarify the puzzle regarding why thermodynamically stable structures are susceptible to shear banding, we consider clusters with a high degree of L5FS in MG as the reinforced particles. We thus line up the heterogeneous structure, STZ activation, and shear banding to give a comprehensive understanding of the shear banding mechanism in MGs. Our work unveils that it is the structural heterogeneity, or, more specifically, the strain gradient that governs the very different deformation behaviors of MGs with different thermodynamic statuses. The strain gradient drives the coalescence of the existing activated regions, which eventually percolate and form shear bands. A high strain gradient means intense but localized deformation, while a small strain gradient means widely and homogenously distributed plastic events. Thus, the higher the strain gradient, the more localized deformation appears. These physical insights may help to understand the essence of the shear banding phenomenon in MGs down to the atomic scale and may be beneficial in designing more reliable amorphous structures to avoid catastrophic failures in real applications.

\section{ACKNOWLEDGMENTS}

We acknowledge insightful discussions with Prof. E. Ma, A. Lemaitre, and C. S. O'Hern. This work is financially supported by the NSFC (No. 11472287, No. 11402269, No. 11672299, and No. 11572324), the National Key Research and Development Program of China (No. 2017YFB0702003), the Key Research Program of Frontier Sciences (Grant No. QYZDJSSW-JSC011), and the Strategic Priority Research Program of the Chinese Academy of Sciences (Grant No. XDB22040302). 
[1] A. Fall, F. Bertrand, G. Ovarlez, and D. Bonn, Phys. Rev. Lett. 103, 178301 (2009).

[2] Y. L. Bai and B. Dodd, Adiabatic Shear Localization: Occurrence, Theories, and Applications (Pergamon, Oxford, UK, 1992).

[3] A. L. Greer, Y. Q. Cheng, and E. Ma, Mater. Sci. Eng. Rep. 74, 71 (2013).

[4] Y. Q. Cheng, E. Ma, and H. W. Sheng, Phys. Rev. Lett. 102, 245501 (2009).

[5] A. Hirata, P. F. Guan, T. Fujita, Y. Hirotsu, A. Inoue, A. R. Yavari, T. Sakurai, and M. W. Chen, Nat. Mater. 10, 28 (2011).

[6] W. H. Wang, Nat. Mater. 11, 275 (2012).

[7] Z. Liu, R. Li, G. Wang, S. Wu, X. Lu, and T. Zhang, Acta Mater. 59, 7416 (2011).

[8] H. W. Sheng, W. K. Luo, F. M. Alamgir, J. M. Bai, and E. Ma, Nature (London) 439, 419 (2006).

[9] D. Ma, A. D. Stoica, and X. L. Wang, Nat. Mater. 8, 30 (2008).

[10] W. K. Luo, H. W. Sheng, F. M. Alamgir, J. M. Bai, J. H. He, and E. Ma, Phys. Rev. Lett. 92, 145502 (2004).

[11] Y. H. Liu, D. Wang, K. Nakajima, W. Zhang, A. Hirata, T. Nishi, A. Inoue, and M. W. Chen, Phys. Rev. Lett. 106, 125504 (2011).

[12] W. Dmowski, T. Iwashita, C. P. Chuang, J. Almer, and T. Egami, Phys. Rev. Lett. 105, 205502 (2010).

[13] J. C. Ye, J. Lu, C. T. Liu, Q. Wang, and Y. Yang, Nat. Mater. 9, 619 (2010).

[14] L. S. Huo, J. F. Zeng, W. H. Wang, C. T. Liu, and Y. Yang, Acta Mater. 61, 4329 (2013).

[15] T. Fujita, K. Konno, W. Zhang, V. Kumar, M. Matsuura, A. Inoue, T. Sakurai, and M. W. Chen, Phys. Rev. Lett. 103, 075502 (2009).

[16] Y. H. Liu, G. Wang, R. J. Wang, D. Q. Zhao, M. X. Pan, and W. H. Wang, Science 315, 1385 (2007).

[17] Y. Q. Cheng and E. Ma, Prog. Mater. Sci. 56, 379 (2011).

[18] J. Ding, S. Patinet, M. L. Falk, Y. Cheng, and E. Ma, Proc. Natl. Acad. Sci. U.S.A. 111, 14052 (2014).

[19] E. Ma, Nat. Mater. 14, 547 (2015).

[20] M. J. Demkowicz and A. S. Argon, Phys. Rev. Lett. 93, 025505 (2004).

[21] M. J. Demkowicz and A. S. Argon, Phys. Rev. B 72, 245206 (2005).

[22] M. J. Demkowicz and A. S. Argon, Phys. Rev. B 72, 245205 (2005).

[23] A. S. Argon, Acta Metall. 27, 47 (1979).

[24] M. L. Falk and J. S. Langer, Phys. Rev. E 57, 7192 (1998).

[25] A. Lemaître, Phys. Rev. Lett. 89, 064303 (2002).

[26] J. Chattoraj and A. Lemaître, Phys. Rev. Lett. 111, 066001 (2013).

[27] S. Ogata, F. Shimizu, J. Li, M. Wakeda, and Y. Shibutani, Intermetallics 14, 1033 (2006).

[28] P. G. Debenedetti and F. H. Stillinger, Nature (London) 410, 259 (2001).

[29] W. L. Johnson, M. D. Demetriou, J. S. Harmon, M. L. Lind, and K. Samwer, MRS Bull. 32, 644 (2007).

[30] Y. Fan, T. Iwashita, and T. Egami, Nat. Commun. 5, 5083 (2014).

[31] Y. Fan, T. Iwashita, and T. Egami, Phys. Rev. Lett. 115, 045501 (2015).
[32] D. Rodney and C. Schuh, Phys. Rev. Lett. 102, 235503 (2009).

[33] F. Spaepen, Acta Metall. 25, 407 (1977).

[34] M. D. Demetriou, J. S. Harmon, M. Tao, G. Duan, K. Samwer, and W. L. Johnson, Phys. Rev. Lett. 97, 065502 (2006).

[35] Z. Lu, W. Jiao, W. H. Wang, and H. Y. Bai, Phys. Rev. Lett. 113, 045501 (2014).

[36] Y. F. Shi and M. L. Falk, Phys. Rev. Lett. 95, 095502 (2005).

[37] G. Voronoi, Journal für die reine und angewandte Mathematik 134, 198 (1908).

[38] H. Jonsson and H. C. Andersen, Phys. Rev. Lett. 60, 2295 (1988)

[39] F. Spaepen, Nature (London) 408, 781 (2000).

[40] H. L. Peng, M. Z. Li, and W. H. Wang, Phys. Rev. Lett. 106, 135503 (2011).

[41] Y. C. Hu, F. X. Li, M. Z. Li, H. Y. Bai, and W. H. Wang, Nat Commun. 6, 8310 (2015).

[42] Z. Wang, B. A. Sun, H. Y. Bai, and W. H. Wang, Nat. Commun. 5, 5823 (2014).

[43] A. J. Cao, Y. Q. Cheng, and E. Ma, Acta Mater. 57, 5146 (2009).

[44] Y. Q. Cheng, A. J. Cao, H. W. Sheng, and E. Ma, Acta Mater. 56, 5263 (2008).

[45] Y. D. Wei, P. Peng, Z. Z. Yan, L. T. Kong, Z. A. Tian, K. J. Dong, and R. S. Liu, Comput. Mater. Sci. 123, 214 (2016).

[46] L. H. Dai, L. F. Liu, and Y. L. Bai, Int. J. Solids Struct. 41, 5979 (2004).

[47] L.-T. To, D. J. Daley, and Z. H. Stachurski, Solid State Sci. 8, 868 (2006).

[48] X. Tong, G. Wang, Z. H. Stachurski, J. Bednarčík, N. Mattern, Q. J. Zhai, and J. Eckert, Sci. Rep. 6, 30876 (2016).

[49] A. C. Eringen, Nonlocal Continuum Field Theories (Springer, Berlin, 2002).

[50] M. F. Ashby, Philos. Mag. 21, 399 (1970).

[51] Z. Ling, L. Luo, and B. Dodd, J. Phys. IV 04, C8-453 (1994).

[52] L. H. Dai, Z. Ling, and Y. L. Bai, Scr. Mater. 41, 245 (1999).

[53] W. D. Nix and H. Gao, J. Mech. Phys. Solids 46, 411 (1998).

[54] H. Gao, Y. Huang, W. D. Nix, and J. W. Hutchinson, J. Mech. Phys. Solids 47, 1239 (1999).

[55] Y. Huang, H. Gao, W. D. Nix, and J. W. Hutchinson, J. Mech. Phys. Solids 48, 99 (2000).

[56] F. K. Yan, N. R. Tao, F. Archie, I. Gutierrez-Urrutia, D. Raabe, and K. Lu, Acta Mater. 81, 487 (2014).

[57] S. Plimpton, J. Comput. Phys. 117, 1 (1995).

[58] M. I. Mendelev, D. J. Sordelet, and M. J. Kramer, J. Appl. Phys. 102, 043501 (2007).

[59] F. Shimizu, S. Ogata, and J. Li, Mater. Trans. 48, 2923 (2007).

[60] See Supplemental Material at http://link.aps.org/supplemental/ 10.1103/PhysRevB.96.094103 for the evolution of other symmetries, radical distribution function of four cooling rate samples, the compression results, a schematic description of the strain gradient effects, as well as details of the mechanical model.

[61] B. Wilner, J. Mech. Phys. Solids 36, 141 (1988).

[62] W. L. Johnson and K. Samwer, Phys. Rev. Lett. 95, 195501 (2005).

[63] V. Hieronymus-Schmidt, H. Rösner, G. Wilde, and A. Zaccone, Phys. Rev. B 95, 134111 (2017).

[64] C. Toninelli, M. Wyart, L. Berthier, G. Biroli, and J.-P. Bouchaud, Phys. Rev. E 71, 041505 (2005). 
[65] N. A. Fleck, G. M. Muller, M. F. Ashby, and J. W. Hutchinson, Acta Metall. 42, 475 (1994).

[66] Y. L. Bai, J. Mech. Phys. Solids 30, 195 (1982).

[67] C. Fressengeas and A. Molinari, J. Mech. Phys. Solids 35, 185 (1987).

[68] J. S. Langer, Phys. Rev. E 70, 041502 (2004).

[69] E. Bouchbinder and J. Langer, Phys. Rev. E 80, 031132 (2009).

[70] I. K. Ono, C. S. O’Hern, D. J. Durian, S. A. Langer, A. J. Liu, and S. R. Nagel, Phys. Rev. Lett. 89, 095703 (2002).

[71] Y. F. Shi, M. B. Katz, H. Li, and M. L. Falk, Phys. Rev. Lett. 98, 185505 (2007).
[72] M. Manning, J. S. Langer, and J. Carlson, Phys. Rev. E 76, 056106 (2007).

[73] E. G. Daub and J. M. Carlson, Phys. Rev. E 80, 066113 (2009).

[74] M. Manning, E. Daub, J. Langer, and J. Carlson, Phys. Rev. E 79, 016110 (2009).

[75] B. Budiansky, J. Mech. Phys. Solids 13, 223 (1965).

[76] R. Hill, J. Mech. Phys. Solids 13, 213 (1965).

[77] M. Q. Jiang and L. H. Dai, J. Mech. Phys. Solids 57, 1267 (2009).

[78] R. Huang, Z. Suo, J. H. Prevost, and W. D. Nix, J. Mech. Phys. Solids 50, 1011 (2002). 